\title{
NOS cofactor tetrahydrobiopterin contributes to anesthetic preconditioning induced myocardial protection in the isolated ex vivo rat heart
}

\author{
CHEN WANG ${ }^{1,2}$, SHIGANG QIAO ${ }^{1,2}$, LEI HONG $^{1}$, JIE SUN ${ }^{1,3}$, TUANJIE CHE $^{4}$, \\ JIANZHONG AN ${ }^{1}$ and AMADOU K.S. CAMARA ${ }^{5}$
}

\begin{abstract}
${ }^{1}$ Institute of Clinical Medicine Research, Departments of ${ }^{2}$ Anesthesiology and Perioperative Medicine, ${ }^{3}$ Gastroenterology and
${ }^{4}$ Laboratory of Precision Medicine and Translational Medicine, The Affiliated Suzhou Science and Technology Town Hospital of Nanjing Medical University, Suzhou, Jiangsu 215153, P.R. China; ${ }^{5}$ Department of Anesthesiology and Physiology,
\end{abstract}

Medical College of Wisconsin, Milwaukee, WI 53226, USA

Received July 4, 2019; Accepted December 4, 2019

DOI: $10.3892 / \mathrm{ijmm} .2019 .4445$

\begin{abstract}
Anesthetic preconditioning (APC) may decrease the myocardium injury nearly $50 \%$ following ischemia/reperfusion (I/R) by enhancing recovery of cardiac function, reducing myocardial enzyme release and lowering infarct size when utilized as pretreatment or posttreatment agents. I/R increases nitric oxide (NO) production through endothelial NO synthase (NOS3) and heat shock protein 90 (HSP90). The present study aimed to observe the role of $\mathrm{BH} 4$ availability and the association of HSP90 with NOS3 in APC-mediated cardioprotection against I/R injury. Isolated rat hearts were subjected to no-flow ischemia for $30 \mathrm{~min}$ and reperfusion for $120 \mathrm{~min}$. Sevoflurane (3.5\%) was administered for $15 \mathrm{~min}$ followed by a $15 \mathrm{~min}$ washout prior to ischemia. 2,4-Diamino6-hydroxypyrimidine (DAHP) or sepiapterin (SP) was administered for $40 \mathrm{~min}$ until the onset of ischemia. The results revealed that compared with pre-ischemic basal levels, $\mathrm{BH} 4$ levels decreased and $\mathrm{BH} 2$ levels increased following I/R. $\mathrm{BH} 4$ levels were significantly increased and $\mathrm{BH} 2$ levels were significantly decreased in the APC $+\mathrm{I} / \mathrm{R}$ hearts compared with the I/R group hearts. The $\mathrm{BH} 4: \mathrm{BH} 2$ ratio in the APC-treated hearts was also increased compared with that in the I/R group hearts. SP increased the recovery of contractile function
\end{abstract}

Correspondence to: Professor Jianzhong An, Institute of Clinical Medicine Research, The Affiliated Suzhou Science and Technology Town Hospital of Nanjing Medical University, 1 Lijiang Road, Suzhou, Jiangsu 215153, P.R. China

E-mail: szkjcyy_ajz@126.com

Professor Amadou K.S. Camara, Department of Anesthesiology and Physiology, Medical College of Wisconsin, 8701 Watertown Plank Road M4280, Milwaukee, WI 53226, USA

E-mail: aksc@mcw.edu

Key words: anesthetics preconditioning, heart, ischemia reperfusion injury, nitric oxide synthase, tetrahydrobiopterin and the production of NO, and decreased the production of superoxide anion $\left(\mathrm{O}_{2} \cdot^{-}\right)$in $\mathrm{I} / \mathrm{R}$ heart, but did not elicit these effects in APC-treated hearts. DAHP treatment inhibited the APC-mediated recovery of contractile function, increased $\mathrm{O}_{2}{ }^{-}$levels and decreased NO production, but had no effect in $\mathrm{I} / \mathrm{R}$ hearts. The cardioprotection of APC was demonstrated to be modulated by the $\mathrm{BH} 4$ precursor $\mathrm{SP}$, which increased $\mathrm{BH} 4$ levels, or DAHP, which inhibited GTP cyclohydrolase I. Both APC and SP treatments increased the combination of HSP90 and NOS3, which improved the NOS3 activity and function. The results suggested that $\mathrm{BH} 4$, which servesas a cofactor for NOS, mediated the resistance of APC to I/R injury by promoting the binding of HSP90 and NOS3.

\section{Introduction}

Nitric oxide (NO) synthase, endothelial (NOS3)-derived NO is an important signaling molecule in the vascular system (1-3). The bioavailability of tetrahydrobiopterin (BH4) is critical for the catalytic activity of NOS3. The decreased effectiveness of BH4 leads to the decoupling of NOS3 and the production of reactive oxygen species (ROS) in endothelial cells $(4,5)$. Decreased bioavailability of NO is an important factor in myocardial ischemia-reperfusion injury. The mechanism of NO reduction is associated with the lack of NOS substrate L-arginine and the cofactor $\mathrm{BH} 4$ leading to NOS decoupling (6).

Ischemia time-dependently decreases cardiac $\mathrm{BH} 4$ content and NOS3 activity, and increases NOS-derived superoxide anion $\left(\mathrm{O}_{2} \cdot^{-}\right)$production, which is considered to contribute to post-ischemic endothelial dysfunction and myocardial injury (6). The decrease in BH4 bioavailability is associated with the uncoupling of NOS3 activity and the production of NOS3-dependent $\mathrm{O}_{2}{ }^{-}$. Increased resistance of the heart to ischemia is associated with the combination of heat shock protein 90 (HSP90) and NOS3 and subsequent increases in NO production (7). BH4 supplementation may restore endothelium-dependent coronary blood flow and decrease ischemia/reperfusion $(\mathrm{I} / \mathrm{R})$ injury in rat hearts $(8,9)$, while 
inhibition of BH4 synthesis may increase I/R injury (10). These results suggest that myocardial $\mathrm{BH} 4$ bioavailability is an important factor in cardioprotection and supports the hypothesis that BH4 may be a novel therapeutic target for the treatment of I/R injury.

Increasing evidence indicates that NOS3-derived NO is a critical mediator of anesthetic preconditioning (APC) (10-13). Under hyperglycemic conditions, the cardioprotective effects of volatile anesthetics by BH4 and HSP90-regulated NOS3 activity was abrogated (14). However, the role of BH4 availability and the association of HSP90 with NOS3 in APC-mediated cardioprotection against $\mathrm{I} / \mathrm{R}$ injury remain to be elucidated. The aims of the present study were to determine: i) Whether BH4 levels were different in I/R rat hearts with or without APC, and whether increasedBH4 levels contributed to resistance to I/R injury by APC; ii) whether BH4 supplementation with $\mathrm{BH} 4$ precursorsepiapterin (SP) and GTP cyclohydrolase I (GCH-1) inhibitor, 2,4-diamino-6-hydroxypyrimidine (DAHP) differentially modulated resistance to $\mathrm{I} / \mathrm{R}$ injury in the control (I/R alone) and APC rat hearts and iii) whether $\mathrm{BH} 4$ supplementation alteredthe $\mathrm{BH} 4: \mathrm{BH} 2$ ratio, consequently affecting NOS3 activity and the association of HSP90 with NOS3 in APC-induced cardioprotection.

\section{Materials and methods}

Animals. The present study was approved by the Institutional Animal Care and Use Committee of Nanjing University. Male Sprague-Dawley rats $(250 \pm 50 \mathrm{~g})$, aged 8-10 weeks, were purchased from the Animal Center of Suzhou University and were housed at $25^{\circ} \mathrm{C}$ with $60 \%$ humidity in a $12: 12 \mathrm{~h}$ light: Dark cycle. All rats were housed in each cage and were allowed ad libitum access to food and water. All procedures performed on the rats used in the present study were in accordance with the National Institute Health Guide for the care and Use of Laboratory Animals.

Isolated heart preparation. The isolated heart preparation was performed as previously described $(8,13,15)$. Briefly, rats were anesthetized with sodium pentobarbital $(50 \mathrm{mg} / \mathrm{kg}$ i.p.) and decapitated when unresponsive to noxious stimulation (pinching the paw). The hearts were excised and perfused in the Langendorff mode at a perfusion pressure equivalent to $80 \mathrm{mmHg}$. Perfusate and bath temperatures were maintained at $37.2 \pm 0.1^{\circ} \mathrm{C}$ using a thermostatically controlled water circulator (Lauda E100; LAUDA). Left ventricular pressure (LVP) was measured isovolumetrically with a transducer connected to a thin saline-filled latex balloon inserted into the left ventricle through the mitral valve from an incision in the left atrium. The hearts were immersed in gassed physiological buffer solution at $37.2^{\circ} \mathrm{C}$, and subjected to $30 \mathrm{~min}$ global ischemia followed by 120 min reperfusion. At the time points prior to ischemia or at the end of the experiments, the hearts were freeze-clamped and stored at $-80^{\circ} \mathrm{C}$ until use in the $\mathrm{BH} 4$ and $\mathrm{BH} 2$ analyses by high performance liquid chromatography (HPLC) or western blot analysis.

Experimental protocols. Each experimental treatment phase lasted for $220 \mathrm{~min}$. A total of 70 rats were used. The functional parameters were stabilized for $30 \mathrm{~min}$; after that the hearts were randomly divided into 7 groups with 10 hearts in each group (Fig. 1). Untreated time controls (TC; without I/R) were perfused for $220 \mathrm{~min}$ without drugs or ischemia (timeline A). After 40 min of vehicle perfusion, the I/R group was subjected to $30 \mathrm{~min}$ of global ischemia and $120 \mathrm{~min}$ of reperfusion; the APC group was exposed to sevoflurane (3.5\%; Abbott Pharmaceutical Co., Ltd.) for $15 \mathrm{~min}$ followed by a $15 \mathrm{~min}$ washout prior to the onset of ischemia (timeline B). In the $\mathrm{SP}+\mathrm{I} / \mathrm{R}$ or SP + APC groups, SP $(50 \mu \mathrm{M}$; cat. no. 11.225; Schircks Laboratories) was administered for $40 \mathrm{~min}$ prior to the onset of ischemia (timeline C). In the DAHP + I/R or DAHP + APC groups, DAHP (2.5 mM; cat. no. D19206; Sigma-Aldrich, Merck KGaA) was administered for $40 \mathrm{~min}$ prior to the onset of ischemia (timeline D).

Measurement of $\mathrm{BH} 4$ and $\mathrm{BH} 2$. The contents of $\mathrm{BH} 4$ and $\mathrm{BH} 2$ in all cardiac homogenates were determined viaHPLC as previously described (8). $\mathrm{BH} 4$ and $\mathrm{BH} 2$ were quantified via HPLC with an electrochemical detector (ESA Biosciences CoulArray ${ }^{\circledR}$ system Model 542) using a Synergi Polar-RP column (Phenomenex) eluted with argon degassed $50 \mathrm{mM}$ phosphate buffer (pH 2.6). Multi-channel colorimetric detection was set between 0 and $600 \mathrm{mV}$. A separate channel was set at $-250 \mathrm{mV}$ in order to verify the reversibility of $\mathrm{BH} 4$ oxidative peak detection. The peak areas were collected at 0 and $150 \mathrm{mV}$ for $\mathrm{BH} 4$, and 280 and $365 \mathrm{mV}$ for $\mathrm{BH} 2$, and the data were combined to obtain a calibration curve. Intracellular concentrations of $\mathrm{BH} 4$ and $\mathrm{BH} 2$ were calculated using authentic external $\mathrm{BH} 4$ and $\mathrm{BH} 2$ standards as previously described (8). Cellular BH4 and BH2 levels were then normalized to cell protein concentrations.

Western blot analysis. The western blot analysiswas performed as previously described (8). The protein content of the samples was determined using a BCA assay kit (P0010; Biyuntian Biotechnology Company) and was adjusted to the same concentration. After denaturation, $20 \mu \mathrm{g}$ of each sample was dissolved in Laemmli sample buffer (cat. no. S3401; Sigma-Aldrich, Merck KGaA) and separated via SDS-PAGE (12\% gel). Following transfer to nitrocellulose membranes, membranes were blocked with 5\% non-fat milk in PBS and probed with primary antibodies at $4^{\circ} \mathrm{C}$ overnight and were incubated with HRP-Conjugated goat anti-rabbit secondary antibodies (1:10,000; cat. no. ab6721; Abcam) or HRP-conjugated goat anti-mouse secondary antibody (1:10,000; cat. no. sc-2031; Santa Cruz Biotechnology, Inc.) for $1 \mathrm{~h}$ at room temperature. The protein bands were visualized with a SuperSignal West Pico kit (cat. no. 34577; Pierce; Thermo Fisher Scientific, Inc.) and band densities were quantified using UN-SCAN-IT software (v.7.0; Silk Scientific, Inc.). Antibodies specific for GCH-1 (cat. no. ab26439, 1:2,000) and GAPDH (cat.no.ab9485, 1:2,500) used in the study were purchased from Abcam. The NOS3 (cat. no. PA3-031A; 1:1,000) and HSP90 (cat. no. MA1-10372; 1:1,000) antibodies were purchased from Invitrogen; Thermo Fisher Scientific, Inc.

Immunoprecipitation assay. Immunoprecipitation was performed as previously described (8). Hearts were homogenized in ice-cold lysis buffer and subjected to SDS-PAGE (12\% gel). Proteins were detected using enhanced chemiluminescence 


\begin{tabular}{rl|l|l|l|}
\multicolumn{1}{c}{$30 \mathrm{~min}$} & \multicolumn{1}{c}{$40 \mathrm{~min}$} & \multicolumn{1}{c}{$30 \mathrm{~min}$} & $120 \mathrm{~min}$ \\
\cline { 2 - 5 } $\begin{array}{r}\text { Time } \\
\text { control }\end{array}$ & Baseline & & & \\
\cline { 2 - 5 } & & & \\
\hline
\end{tabular}

B

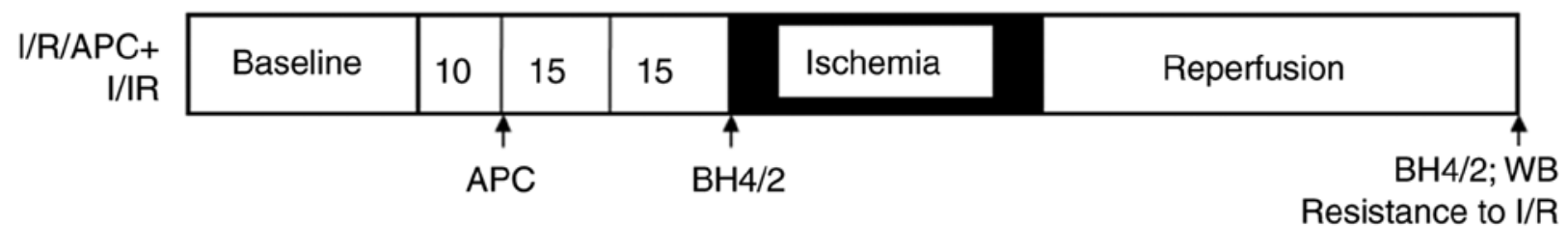

C

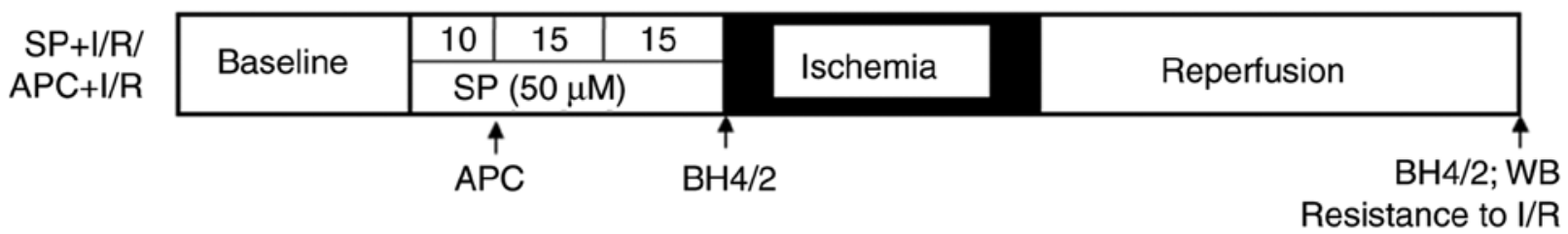

D $D A H P+1 / R /$ $\mathrm{APC}+\mathrm{I} / \mathrm{R}$
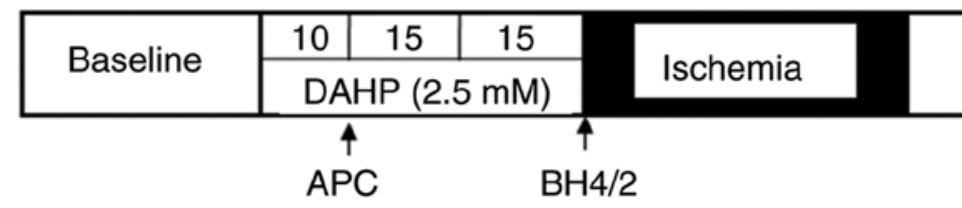

Reperfusion

$\mathrm{BH} 4 / 2$; WB

Resistance to $\mathrm{I} / \mathrm{R}$

Figure 1. Schematic illustration of the experimental protocols used in all groups. (A) Timeline A represents the untreated time controls (TC, no I/R). (B) Timeline B represents the ischemia reperfusion controls (I/R) or I/R with sevoflurane treatment (APC + I/R). (C) Timeline C represents I/R with SP treatment $(\mathrm{SP}+\mathrm{I} / \mathrm{R})$ or I/R with SP and sevoflurane treatment $(\mathrm{SP}+\mathrm{APC}+\mathrm{I} / \mathrm{R})(\mathrm{D})$ Time line D represents I/R with DAHP treatment (DAHP + I/R) or I/R with DAHP and sevoflurane treatment (DAHP + APC + I/R). BH4/2 marks denote the time points at which the tissue samples were harvested for the HPLC assay for BH4 and BH2. Resistance to I/R injury means determining functional recovery after 30 min global ischemia and 120 min reperfusion. The APC group received sevoflurane (3.5\%) for $15 \mathrm{~min}$ followed by a $15 \mathrm{~min}$ washout before global ischemia. Vehicle, SP or DAHP were perfused for $40 \mathrm{~min}$ prior to ischemia in the presence of absence of APC. I/R, ischemia/reperfusion; APC, anesthetic preconditioning; SP, sepiapterin; DAHP, 2,4-diamino-6-hydroxypyrimidine.

detection reagents (cat. no. 34577; Pierce; Thermo Fisher Scientific, Inc.) for densitometric analysis with UN-SCAN-IT software.

Reactive oxygen species $(R O S)$ detection. $\mathrm{ROS}\left(\mathrm{O}_{2} \cdot-\right)$ production was detected using lucigenin-enhanced chemiluminescence as previously described (8). During the first minute of reperfusion, coronary effluent $(1 \mathrm{ml})$ was collected and $500 \mu \mathrm{l}$ was immediately transferred to a $1.5 \mathrm{ml}$ vial, in which $5 \mu 1$ of $500 \mu \mathrm{M}$ lucigenin (cat. no. M8010; Sigma-Aldrich; Merck $\mathrm{KGaA}$ ) was added. The final concentration was $5 \mu \mathrm{M}$. The vial was placed in a luminometer (Turner BioSystems, Inc.) in order to measure chemiluminescence for $5 \mathrm{~min}$ in the dark. A vial containing lucigenin was used to measure background luminescence, and this background value was subtracted from each sample value.The data of $\mathrm{O}_{2} \cdot-$ production was expressed as relative light units.

NO detection. In order to assess the effects of $\mathrm{BH} 4$ on NOS3 coupling, at $1 \mathrm{~min}$ of reperfusion, $1 \mathrm{ml}$ coronary effluent was collected and NO concentrations were immediately determined using a NO electrode (World Precision Instruments). The values were normalized by heart wet weight and coronary flow rate as previously described (8). The NO electrodes were calibrated using $\mathrm{NaNO}_{2}$ (cat. no. 72586; Sigma-Aldrich; Merck $\mathrm{KGaA}$ ) and $\mathrm{KI}$ (cat. no. 746428$)+\mathrm{H}_{2} \mathrm{SO}_{4}$ (cat. no. 339741) (both
Sigma-Aldrich; Merck KGaA) following the manufacturer's protocol, to quantify the amount of NO produced.

Statistics analysis. The data are presented as the means \pm standard error of the mean. In order to examine the overall differences between the groups, a two-way analysis of variance was used. The Student-Newman-Keuls multiple comparison post hoc test was used to differentiate within the groups. SPSS 19.0 (IBM Corp.) was used to perform the statistical analysis. $\mathrm{P}<0.05$ was considered to indicate a statistically significant difference.

\section{Results}

$B H 4$ and $B H 2$ levels in $A P C$ and $I / R$ rat hearts. As presented in Fig. 2, prior to ischemia, $\mathrm{BH} 4$ and $\mathrm{BH} 2$ levels were similar in both the I/R and APC $+\mathrm{I} / \mathrm{R}$ groups (Fig. $2 \mathrm{~A}$ ). The BH2 level was significantly increased and the BH4 level decreased significantly in $\mathrm{I} / \mathrm{R}$ hearts when compared with the pre-ischemia baseline values. Following I/R, the BH4 levels $(27.4 \pm 1.9$ vs. $18.1 \pm 1.9 \mathrm{pmols} / \mathrm{mg}$ protein) were increased and $\mathrm{BH} 2$ levels ( $22.7 \pm 1.8$ vs. $40.3 \pm 3.2 \mathrm{pmols} / \mathrm{mg}$ protein) were decreased in the $\mathrm{APC}+\mathrm{I} / \mathrm{R}$ hearts when compared with the $\mathrm{I} / \mathrm{R}$ hearts $(\mathrm{P}<0.05$; $\mathrm{n}=10$ /group). The $\mathrm{BH} 4: \mathrm{BH} 2$ ratio in $\mathrm{APC}+\mathrm{I} / \mathrm{R}$ rat hearts was increased 2 -fold compared with the I/R hearts $(1.2 \pm 0.15$ vs. $0.45 \pm 0.09$ ). 
A
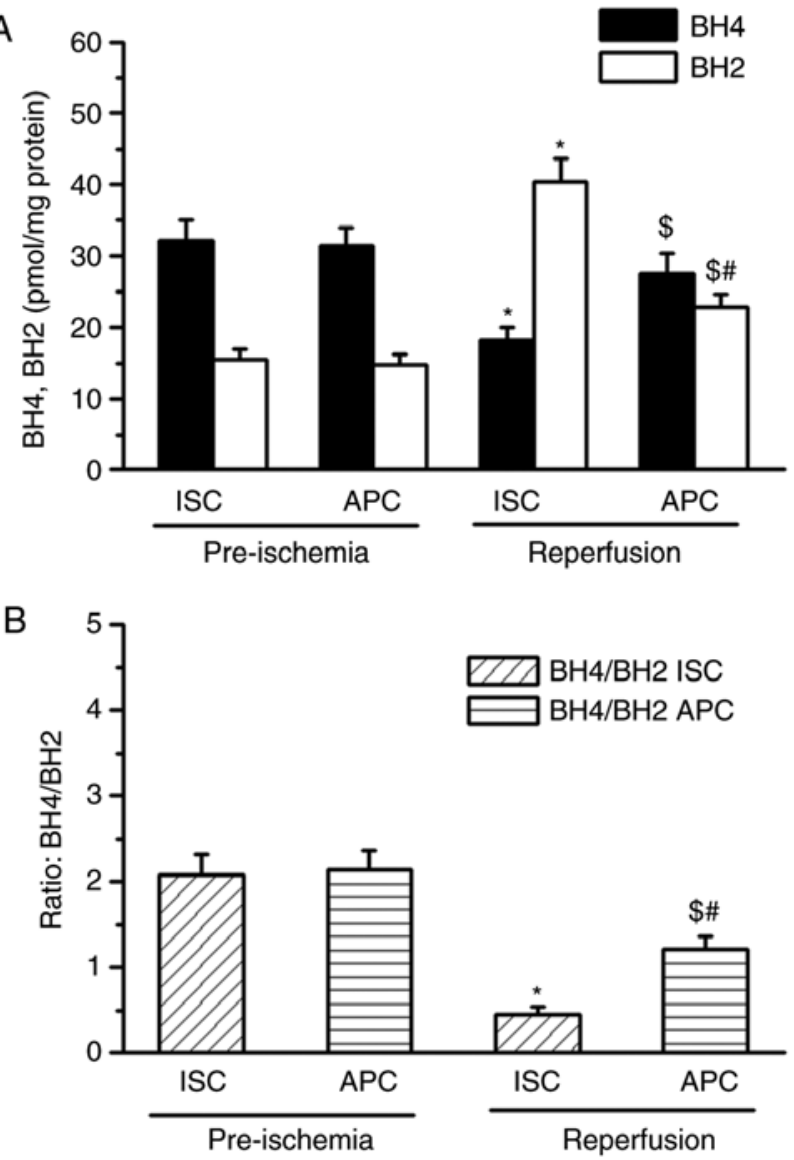

Figure 2. $\mathrm{BH} 4, \mathrm{BH} 2$ levels and the ratio of $\mathrm{BH} 4 / \mathrm{BH} 2$ in rat hearts. (A) $\mathrm{BH} 4$ and $\mathrm{BH} 2$ levels and (B) BH4:BH2 ratio in the TC, APC, I/R and APC + I/R rat hearts. In the TC and APC groups, hearts were harvested after $70 \mathrm{~min}$ perfusion without or with APC treatment. In the I/R and APC $+I / R$ groups, hearts were harvested after $30 \mathrm{~min}$ no-flow global ischemia and $120 \mathrm{~min}$ reperfusion. The data are presented as mean \pm standard error of the mean. ${ }^{*} \mathrm{P}<0.05$ vs. I/R pre-ischemia group. ${ }^{*} \mathrm{P}<0.05$ vs. $\mathrm{APC}+\mathrm{I} / \mathrm{R}$ pre-ischemia group. ${ }^{\$} \mathrm{P} 0.05$ vs.I/R reperfusion group. $\mathrm{n}=10 /$ group. TC, time control; ISC, ischemia; $\mathrm{I} / \mathrm{R}$, ischemia/reperfusion; APC, anesthetic preconditioning.

GCH-1 protein levels. Fig. 3 presents the GCH-1 contents in TC, I/R and APC + I/R heart tissues. The GCH-1 expression level in APC $+\mathrm{I} / \mathrm{R}$ hearts was increased by almost $30 \%$ compared with that in $\mathrm{I} / \mathrm{R}$ hearts after $\mathrm{I} / \mathrm{R}$ ( $\mathrm{n}=3 /$ group).

Supplementation of SP or DAHP on cardiac function. In order to determine whether $\mathrm{BH} 4$ contributed to the increased resistance to I/R injury in APC + I/R hearts compared with $\mathrm{I} / \mathrm{R}$ hearts, the present study perfused isolated hearts with either a GCH-1 inhibitor (DAHP, $2.5 \mathrm{mM}$ ) or a BH4 donor (SP, $50 \mu \mathrm{M}$ ) for $40 \mathrm{~min}$ prior to ischemia with or without APC treatment. Left ventricular developed pressure (LVDP) was measured after 120 min reperfusion, using the percentage of pre-drug and pre-ischemic values. The recovery rate of LVDP following $\mathrm{I} / \mathrm{R}$ in the APC $+\mathrm{I} / \mathrm{R}$ hearts $(62.7 \pm 7.6 \mathrm{mmHg}$ and $62.6 \pm 5.2 \%)$ was significantly increased compared with that in the $\mathrm{I} / \mathrm{R}$ group $(48.0 \pm 4.0 \mathrm{mmHg}$ and $47.4 \pm 5.1 \%)(\mathrm{P}<0.01)$. SP treatment significantly improved the LVDP in I/R hearts $(64.5 \pm 6.7 \mathrm{mmHg}$ and $64.3 \pm 6.2 \%$ ), but did not increase the recovery of LVDP in APC $+\mathrm{I} / \mathrm{R}$ hearts $(68 \pm 6.1 \mathrm{mmHg}$ and $66 \pm 5.9 \%$; Fig. 4A). DAHP treatment decreased the recovery of
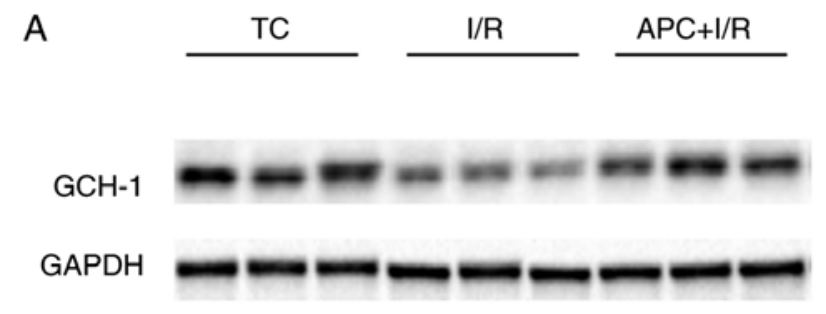

B

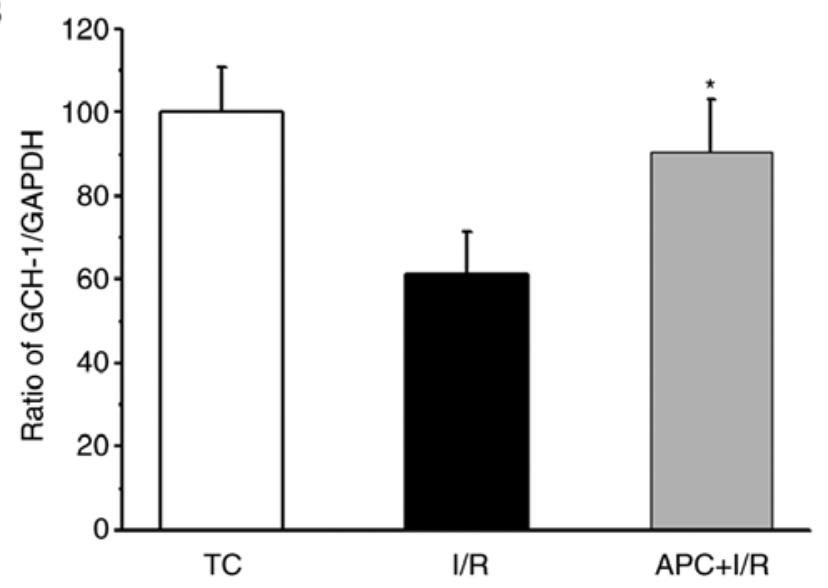

Figure 3. GCH-1 protein levels in the TC, I/R and APC + I/R rat heart homogenates. (A) GCH-1 and GAPDH were detected by western blot analysis using lysates of heart homogenates. GADPH was used as a loading protein control. (B) The densitometric analyses of the blot gel in part A are presented as GCH1/GADPH ratio for each group. Data are presented as the mean \pm standard error of the mean. $n=3 /$ group). ${ }^{*} \mathrm{P}<0.05 \mathrm{vs}$. I/R group. GCH-1, GTP cyclohydrolase I; TC, time control; I/R, ischemia/reperfusion; $\mathrm{APC}$, anesthetic preconditioning.

LVDP in APC hearts $(41.7 \pm 5.1 \mathrm{mmHg}$ and $45.7 \pm 4.6 \%)$ to the values observed in the I/R group hearts (Fig. $4 \mathrm{~B}$ ).

Supplementation of SP or DAHP on cardiac BH4 and BH2 levels. In order to observe the changes in $\mathrm{BH} 4$ and $\mathrm{BH} 2$ levels following SP or DAHP treatment in I/R or APC $+\mathrm{I} / \mathrm{R}$ hearts, $\mathrm{BH} 4$ and $\mathrm{BH} 2$ levels were measured at $40 \mathrm{~min}$ after perfusion with $\mathrm{SP}$ or DAHP (pre-ischemia) or at 120 min reperfusion. Following SP treatment and prior to ischemia in the $\mathrm{SP}+\mathrm{I} / \mathrm{R}$ hearts, the levels of BH4 (45.4 \pm 5.4 vs. $32.1 \pm 2.9$ pmols $/ \mathrm{mg}$ protein; $\mathrm{P}<0.05$ ) and $\mathrm{BH} 2(203 \pm 19$ vs. $15.5 \pm 1.4 \mathrm{pmol} / \mathrm{mg}$ protein $)$ were significantly increased when compared with the I/R hearts $(\mathrm{P}<0.001$; Fig. 5A). At 120 min reperfusion, SP treatment increased BH4 levels significantly in the SP+IR group compared with the $\mathrm{I} / \mathrm{R}$ group $(32.4 \pm 4.5$ vs. $18.1 \pm 1.9 \mathrm{pmol} / \mathrm{mg}$ protein; $\mathrm{P}<0.05)$. In the $\mathrm{APC}+\mathrm{I} / \mathrm{R}$ rat hearts, DAHP treatment prior to ischemia did not affect BH4 or BH2 levels (Fig. 5B). Following I/R, DAHP treatment significantly decreased BH4 levels (21.4 \pm 2.2 vs. $27.4 \pm 2.2$ $\mathrm{pmol} / \mathrm{mg}$ protein) and increased BH2 levels $(56.1 \pm 6.2$ vs. $22.7 \pm 1.8 \mathrm{pmol} / \mathrm{mg}$ protein) in the DAHP+APC hearts compared with the APC $+\mathrm{I} / \mathrm{R}$ hearts $(\mathrm{P}<0.01)$. After $120 \mathrm{~min}$ reperfusion, the $\mathrm{BH} 4: \mathrm{BH} 2$ ratio was decreased in DAHP+APC $+\mathrm{I} / \mathrm{R}$ hearts compared with the APC $+\mathrm{I} / \mathrm{R}$ hearts $(\mathrm{P}<0.01)$.

Supplementation of SP or DAHP on the association of HSP90 and NOS3. In order to observe the effects of SP or APC on the interaction between HSP90 and NOS3 in I/R rat hearts, 
A
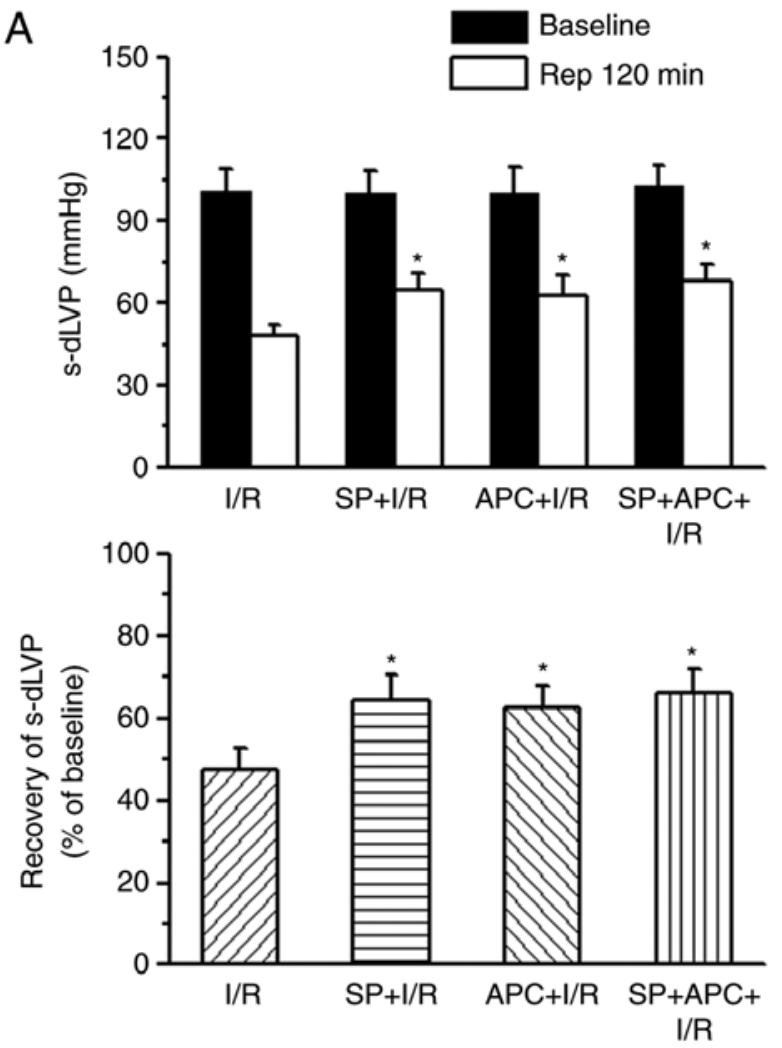

B
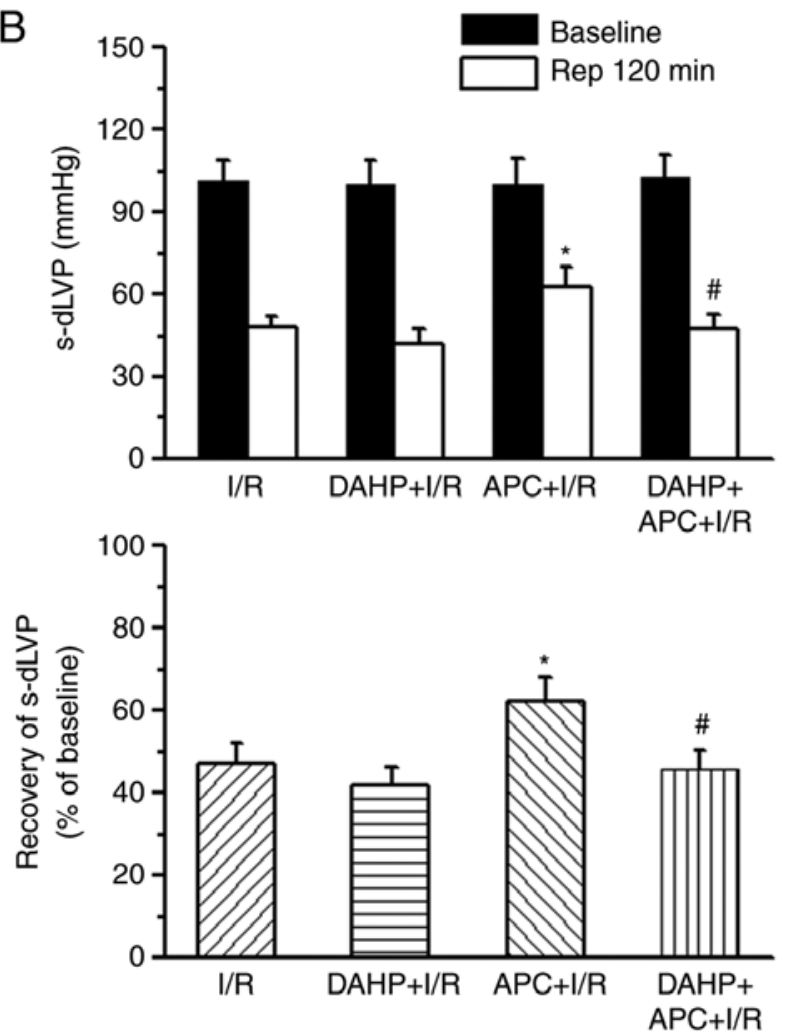

Figure 4. Resistance to myocardial I/R injury in heart tissues from I/R rats treated with SP and APC + I/R rats treated with DAHP. Isolated rat hearts were perfused with (A) SP $(50 \mu \mathrm{M})$ or (B) DAHP $(2.5 \mathrm{mM})$ for $40 \mathrm{~min}$ prior to $30 \mathrm{~min}$ no-flow global ischemia followed by 120 min reperfusion. The upper graphs in parts A and $\mathrm{B}$ demonstrate the absolute values for the s-dLVP $(\mathrm{mmHg})$, and the lower graphs in parts A and B demonstrate the relative recovery of s-dLVP for each group based on their respective basal values. Data are presented as mean \pm standard error of the mean(n=10/group). ${ }^{*} \mathrm{P}<0.05$ vs. I/R group. ${ }^{\#} \mathrm{P}<0.05 \mathrm{vs}$. APC $+\mathrm{I} / \mathrm{R}$ group. SP, sepiapterin; DAHP, 2,4-diamino-6-hydroxypyrimidine; dLVP, developed left ventricle pressure; s-dLVP, systolic minus diastolic left ventricle pressure.

NOS3 was immunoprecipitated from the homogenates of $\mathrm{I} / \mathrm{R}$ hearts following APC, SP or DAHP treatment and probed for NOS3 and HSP90 proteins (Fig. 6). The results demonstrated an increased association between HSP90 and NOS3 (>30\%) in the SP+IR (IB hsp90-SR+I/R row) and APC + I/R hearts (IB hsp90-APC + I/R row) compared with the I/R group. However, this increase in association betweenHSP90 and NOS3 in the $\mathrm{APC}+\mathrm{I} / \mathrm{R}$ hearts was inhibited by treatment with DAHP (IB hsp90-DAHP+APC + I/R row).

$\mathrm{NO}$ and $\mathrm{O}_{2} \cdot{ }^{-}$levels following $\mathrm{I} / \mathrm{R}$. The present study also measured $\bullet \mathrm{NO}$ and $\mathrm{O}_{2} \cdot{ }^{-}$production in I/R hearts following SP treatment or APC + I/R hearts following DAHP treatment (Fig. 7). The data revealed that the lucigenin chemiluminescence signal intensity, which represents $\mathrm{O}_{2} \cdot^{-}$(ROS) production, was significantly decreased in the $\mathrm{SP}+\mathrm{I} / \mathrm{R}$ and $\mathrm{APC}+\mathrm{I} / \mathrm{R}$ rat hearts, but increased in DAHP $+\mathrm{APC}+\mathrm{I} / \mathrm{R}$ rat hearts at the end of $120 \mathrm{~min}$ reperfusion. Following treatment with SP, the NO levels in the SP $+\mathrm{I} / \mathrm{R}$ group was significantly increased compared with those in the I/R group, whereas DAHP treatment significantly decreased the NO levels in DAHP + APC + I/R hearts. APC treatment also significantly increased NO production in APC $+\mathrm{I} / \mathrm{R}$ hearts compared with the I/R group.

\section{Discussion}

The maintenance of physiological levels of NO produced by NOS3 represents a key element for vascular function $(16,17)$.
As a pteridine cofactor, $\mathrm{BH} 4$ is an important regulator of $\mathrm{NOS} 3$ function, as $\mathrm{BH} 4$ is required to maintain enzymatic coupling of L-arginine oxidation, to produce NO $(18,19)$. The present study assessed the role of BH4 and the BH4 biosynthetic precursor, $\mathrm{SP}$, in the mechanisms underlying resistance to $\mathrm{I} / \mathrm{R}$ injury in $\mathrm{I} / \mathrm{R}$ hearts with or without APC. The results revealed that: i) $\mathrm{BH} 4$ levels were significantly increased and $\mathrm{BH} 2$ levels were significantly decreased in APC $+\mathrm{I} / \mathrm{R}$ hearts compared with the I/R group hearts. The $\mathrm{BH} 4: \mathrm{BH} 2$ ratio in the APC-treated hearts was increased 2-fold compared with that in the I/R group hearts. The recovery of cardiac function in the APC-treated hearts was consistent with the alterations of $\mathrm{BH} 4$ and $\mathrm{BH} 2$ levels, and the $\mathrm{BH} 4: \mathrm{BH} 2$ ratio; ii) $\mathrm{SP}$ supplementation increased the resistance to I/R injury in the I/R group, and GCH-1 inhibition decreased APC-induced cardiac function recovery; iii) SP supplementation increased the association between HSP90 and NOS3; while GCH-1 inhibition by DAHP decreased this association; iv) ROS $\left(\mathrm{O}_{2}{ }^{-}\right)$production decreased and NO levels were increased significantly following SP treatment in the I/R group, and ROS production increased and $\mathrm{NO}$ levels decreased following DAHP treatment in APC $+\mathrm{I} / \mathrm{R}$ hearts at the initial time of reperfusion. The results of the present study indicate that BH4 and the ongoing synthesis of BH4 participated in the susceptibility to I/R injury in the APC $+I / R$ and I/R group hearts. APC-induced resistance to $\mathrm{I} / \mathrm{R}$ injury was mediated by the NOS3 cofactor BH4 and the elevation of HSP90-NOS3 association. 

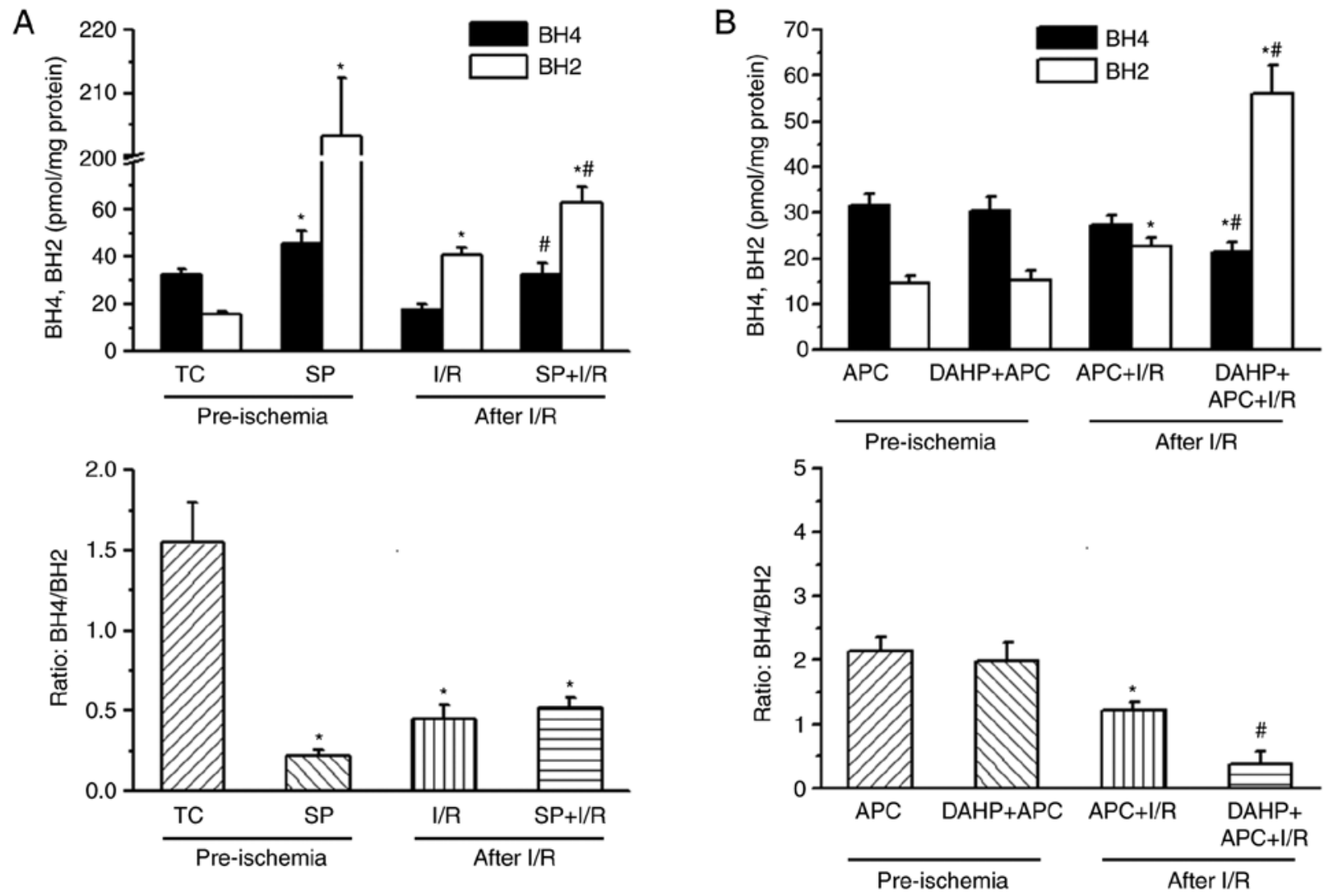

Figure 5. BH4 and $\mathrm{BH} 2$ levels and ratio of $\mathrm{BH} 4 / \mathrm{BH} 2$ in (A) TC and $\mathrm{I} / \mathrm{R}$ rat hearts with or without SP prior to ischemia or following reperfusion (50 $\mu \mathrm{M})$ or (B) APC and APC $+\mathrm{I} / \mathrm{R}$ hearts with or without DAHP $(2.5 \mathrm{mM})$ treatment prior to ischemia or following reperfusion. The values are presented as mean \pm standard error of the mean ( $\mathrm{n}=10$ /group). ${ }^{\mathrm{P}}<0.05$ vs. TC or APC pre-ischemia group. ${ }^{\prime \prime} \mathrm{P}<0.05 \mathrm{vs}$. I/R or APC $+\mathrm{I} / \mathrm{R}$ reperfusion group. TC, time control; I/R, ischemia/reperfusion; APC, anesthetic preconditioning; SP, sepiapterin; DAHP, 2,4-diamino-6-hydroxypyrimidine.

In I/R-injured hearts, lowering BH4 levels may be an important cellular defect involving endothelial and cardiomyocyte dysfunction $(6,20)$. The role of BH4 in NOS activity is important in cardioprotection. The decrease in $\mathrm{BH} 4$ levels not only prevents NOS3 from generating NO, but also causes NOS3 'uncoupling', resulting in NOS3 generating $\mathrm{O}_{2}$. instead of NO (21). BH4 deficiency due to decreased $\mathrm{BH} 4$ biosynthesis or $\mathrm{BH} 4$ oxidation to $\mathrm{BH} 2$ (less catalytic activity) is known to be one of the causes of $I / R$ injury (8). The results of the present study are consistent with these data, in that $\mathrm{BH} 4$ level and the $\mathrm{BH} 4: \mathrm{BH} 2$ ratio in APC-treated hearts were significantly increased compared with that in the I/R group, confirming that $\mathrm{BH} 4$ serves a crucial role in I/R injury. The importance of the $\mathrm{BH} 4: \mathrm{BH} 2$ ratio in determining whether $\mathrm{BH} 4$ and $\mathrm{BH} 2$ bind to NOS3 has been revealed in recent studies. As the affinity of $\mathrm{BH} 4$ and $\mathrm{BH} 2$ to NOS3 is equal, $\mathrm{BH} 4$ in NOS3 can be rapidly and efficiently replaced by $\mathrm{BH} 2$, resulting in the uncoupling of NOS3 activity, and decreased NO synthesis $(2,22)$. APC treatment demonstrated increased $\mathrm{BH} 4$ and decreased $\mathrm{BH} 2$ levels following I/R. Therefore, in addition to decreased $\mathrm{BH} 4$ levels, the $\mathrm{BH} 4: \mathrm{BH} 2$ ratio was significantly decreased in I/R group hearts compared with that in the APC + I/R hearts, which may also lead to NOS3 uncoupling, an increase in $\mathrm{O}_{2}{ }^{--}$production and decreased cardiac function recovery.

Increasing the availability of $\mathrm{BH} 4$ by modulating $\mathrm{GCH}-1$ has been recognized as a novel strategy for protecting the heart during post-infarction remodeling, dilated myopathic remodeling and cardiac hypertrophy (23). Certain studies have demonstrated that the treatment of exogenous $\mathrm{BH} 4$ or BH4 precursor SP may promote NO production and NOS-dependent coronary flow recovery following ischemia $(6,24)$. SP supplementation enhanced the resistance to $\mathrm{I} / \mathrm{R}$ injury in the I/R group. Notably, the results of the present study demonstrated that SP did not alter the APC-induced cardioprotection observed, suggesting that the cardiac protection induced by APC had reached its maximum effect. Resistance to I/R injury by APC was abrogated to levels that were not significantly different from the I/R group when GCH-1 was inhibited with DAHP. In addition, the GCH-1 inhibitor DAHP did not affect the functional recovery of the I/R group, but SP supplementation increased the functional recovery to a level comparable with that of APC-treated hearts. This association provides evidence that $\mathrm{BH} 4$ levels are associated with APC-induced cardioprotection.

The present study hypothesized that $\mathrm{BH} 4$ serves a crucial role in NOS3 activity, $\mathrm{NO}$ generation, potentially with $\mathrm{O}_{2}{ }^{-}$ formation as suggested by the lucigenin chemiluminescence assay, and vascular reactivity during I/R. The increased lucigenin chemiluminescence signal intensity, representing the level of $\mathrm{O}_{2} \cdot-$ released, or net emission, from I/R rat hearts was significantly attenuated by SP and APC, but increased in the $\mathrm{DAHP}+\mathrm{APC}+\mathrm{I} / \mathrm{R}$ group. Furthermore, there was a greater lucigenin chemiluminescence signal intensity from $\mathrm{I} / \mathrm{R}$ alone 
A

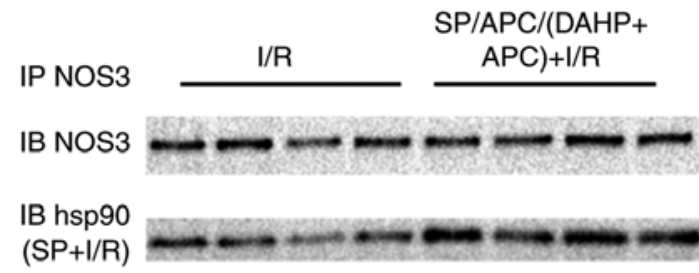

IB hsp90

$(\mathrm{APC}+\mathrm{l} / \mathrm{R})$

IB hsp90

(DAHP+

$\mathrm{APC}+\mathrm{l} / \mathrm{R})$

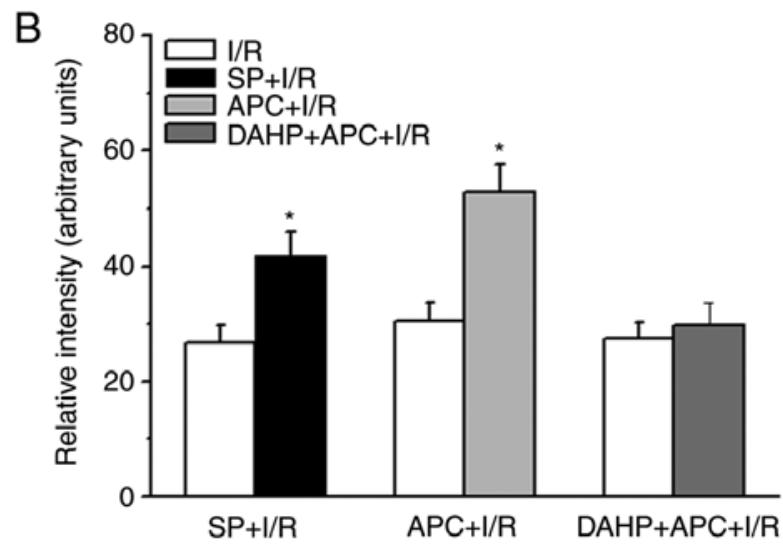

Figure 6. Western blot analysis of the association between NOS3 and HSP90 in heart homogenates from rat hearts. (A) The IB NOS3 row represents the $\mathrm{I} / \mathrm{R}$ and $\mathrm{APC}+\mathrm{I} / \mathrm{R}$ heart tissues. The IB hsp90 (SP + I/R) row represents the I/R and SP + IR heart tissues. The IB hsp90 (APC + I/R) row represents the I/R and APC + IR heart tissues. The IB hsp90 (DAHP + APC + I/R) row represents the I/R and DAHP + APC + I/R heart tissues. The effects of SP $(50 \mu \mathrm{M})$, APC (sevoflurane at $3.5 \%)$ or DAHP $(2.5 \mathrm{mM})$ treatment on HSP90 association with NOS3. (B) The densitometric analyses of the blot gel in part A. $\mathrm{n}=3$ /group. IP, immunoprecipitation; IB, immunoblot. ${ }^{*} \mathrm{P}<0.05$ vs. I/R group. NOS3, nitric oxide synthase, endothelial; HSP90, heat shock protein 90; I/R, ischemia/reperfusion; APC, anesthetic preconditioning; SP sepiapterin; DAHP, 2,4-diamino-6-hydroxypyrimidine.

hearts. NO release was increased in I/R alone hearts through SP supplementation, while DAHP significantly decreased NO production in APC $+\mathrm{I} / \mathrm{R}$ hearts. These data confirmed that BH4 levels were associated with NOS-dependent NO production, and decreased $\mathrm{O}_{2} \cdot-$ generation.

A previous study has demonstrated that APC enhanced GTPCH-1 and NOS3 expression levels, and promoted the production of $\mathrm{NO}$ in the myocardium following reperfusion (11). In C57BL/6 mice hearts, the rate of LV pressure rise, $\mathrm{BH} 4$ level, $\mathrm{Ca}^{2+}$ handling proteins and $\mathrm{SR} \mathrm{Ca}^{2+}$ release were decreased following I/R, which was associated with GCH1 degradation (25). In the coronary arteries of rats with heart failure, both NOS3 and GCH1 protein expression levels increased concomitantly with the decrease in ROS generation, increase of NO bioavailability and NOS3 coupling (26). A high level of $\mathrm{GCH}-1$ expression in $\mathrm{APC}+\mathrm{I} / \mathrm{R}$ rats was also observed in the present study, indicating that increased GCH-1 expression is involved in volatile anesthetic-induced cardioprotection.

Previous studies have demonstrated that an increased association between HSP90 and NOS3 contributed to cardioprotection against $\mathrm{I} / \mathrm{R}$ by increasing $\mathrm{NO}$ generation and
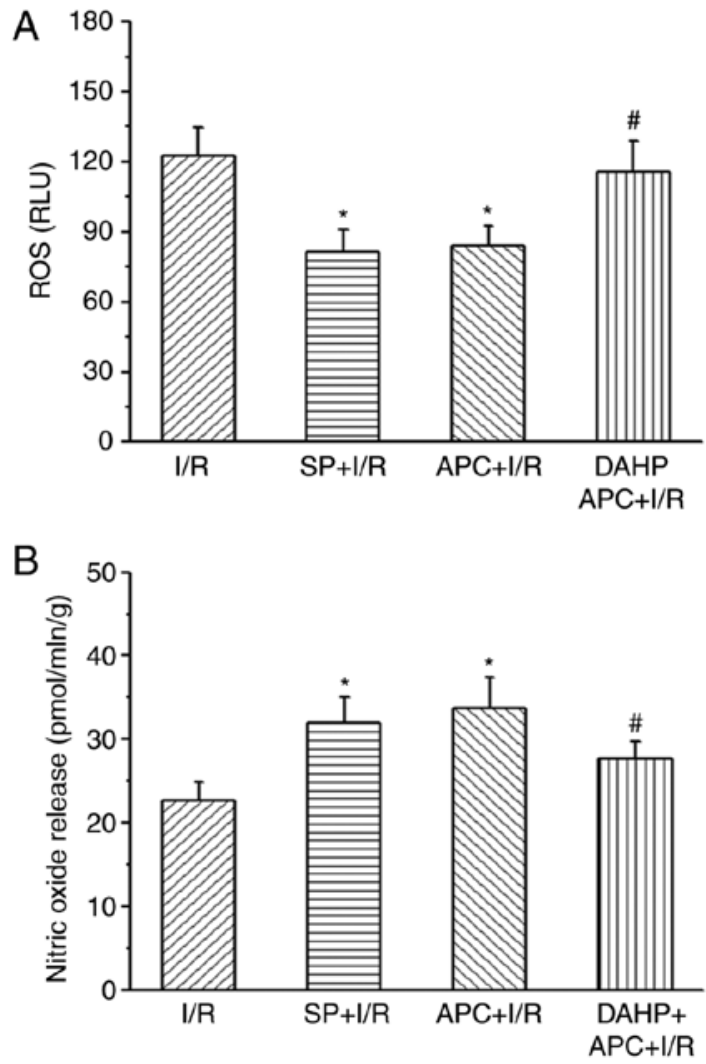

Figure 7. Lucigenin chemiluminescence signal intensity. Superoxide and NO production from hearts of I/R rats with/without SP $(50 \mu \mathrm{M})$ treatment and $\mathrm{APC}+\mathrm{I} / \mathrm{R}$ with/without DAHP $(2.5 \mathrm{mM})$ treatment at $1 \mathrm{~min}$ reperfusion. (A) ROS production in I/R, SP $+\mathrm{I} / \mathrm{R}, \mathrm{APC}+\mathrm{I} / \mathrm{R}$ and $\mathrm{DAHP}+\mathrm{APC}+\mathrm{I} / \mathrm{R}$ groups. (B) NO levels in the I/R, SP $+\mathrm{I} / \mathrm{R}, \mathrm{APC}+\mathrm{I} / \mathrm{R}$ and DAHP $+\mathrm{APC}+\mathrm{I} / \mathrm{R}$ groups. The values are presented as mean \pm standard error of the mean. $\mathrm{n}=6$ /group. ${ }^{*} \mathrm{P}<0.05$ vs. I/R group. ${ }^{\#} \mathrm{P}<0.05$ vs. $\mathrm{I} / \mathrm{R}+\mathrm{APC}$ group. $\mathrm{NO}$, nitric oxide; I/R, ischemia/reperfusion; APC, anesthetic preconditioning; SP, sepiapterin; DAHP, 2,4-diamino-6-hydroxypyrimidine.

decreasing $\mathrm{O}_{2}{ }^{-}$production $(7,27)$. The results of the present study also demonstrated that SP supplementation increased the association between HSP90 and NOS3, while DAHP supplementation decreased this association in APC $+\mathrm{I} / \mathrm{R}$ hearts, consistent with the results of a previous study (8). These data demonstrated that BH4 improved NOS3 activity and function, which is an important mechanism underlying APC-induced cardioprotection.

In summary, the present study demonstrated that BH4 and GCH-1 differentially modulated APC-induced cardioprotection against I/R injury. BH4 biosynthesis, regulated by $\mathrm{GCH}-1$, served a key role in the enhancement of $\mathrm{NO}$ generation during APC, and NO, in turn, conferred a cardioprotective effect. Furthermore, the differences in the $\mathrm{BH} 4: \mathrm{BH} 2$ ratio, which were associated with NOS3 coupling state and the increase in heart BH4 oxidation, conferred a cardioprotective effect in APC-treated hearts. These results indicated that APC may mediate resistance to $\mathrm{I} / \mathrm{R}$ injury by enhancement of $\mathrm{BH} 4$ level and the association between HSP90 and NOS3. In addition, the combination of BH4 and HSP90 that contributes to the resistance to $\mathrm{I} / \mathrm{R}$ injury may be dependent on increasing NOS3-coupled activity and function. Overall, the pharmacological targeting of the BH4-HSP90-NOS3 axis may represent a novel approach to alleviating myocardial I/R injury. 


\section{Acknowledgements}

Not applicable.

\section{Funding}

The present study was supported by the Suzhou Science and Technology Development Plan (grant nos. SS201756 and SS201613); the Suzhou New District Science and Technology Project (grant no. 2017Z004); and the National Science and Technology Development Plan (grant no. NSFC 81703501).

\section{Availability of data and materials}

All data generated or analyzed during the present study are available from the corresponding author on reasonable request.

\section{Authors' contributions}

$\mathrm{CW}, \mathrm{JA}$ and $\mathrm{AC}$ were involved in the experimental design, data collection, data analysis and manuscript writing. SQ, LH, JS and TC performed the experiments. CW, JA and AC provided critical comments throughout the process of the present study. All authors read and approved the final manuscript.

\section{Ethics approval and consent to participate}

The present study was approved by the Institutional Animal Care and Use Committee of Nanjing University

\section{Patient consent for publication}

Not applicable.

\section{Competing interests}

The authors have declared that they have no competing interests.

\section{References}

1. Bredt DS and Snyder SH: Nitric oxide: A physiologic messenger molecule. Annu Rev Biochem 63: 175-195, 1994.

2. Ziolo MT, Kohr MJ and Wang H: Nitric oxide signaling and the regulation of myocardial function. J Mol Cell Cardiol 45: 625-632, 2008

3. Gaynullina DK, Schubert R and Tarasova OS: Changes in endothelial nitric oxide production in systemic vessels during early ontogenesis-A key mechanism for the perinatal adaptation of the circulatory system. Int J Mol Sci 20: pii: E1421, 2019.

4. Yuyun MF, Ng LL and Ng GA: Endothelial dysfunction, endothelial nitric oxide bioavailability, tetrahydrobiopterin, and 5 -methyltetrahydrofolate in cardiovascular disease. Where are we with therapy? Microvasc Res 119: 7-12, 2018.

5. Xie L, Hu D, Qin H, Zhang W, Zhang S, Feng Y, Yao H, Xiao Y, Yao $\mathrm{K}$ and Huang $\mathrm{X}$ : In vivo gum arabic-coated tetrahydrobiopterin protects against myocardial ischemia reperfusion injury by preserving eNOS coupling. Life Sci 219: 294-302, 2019.

6. Dumitrescu C, Biondi R, Xia Y, Cardounel AJ, Druhan LJ, Ambrosio G and Zweier JL: Myocardial ischemia results in tetrahydrobiopterin (BH4) oxidation with impaired endothelial function ameliorated by BH4. Proc Natl Acad Sci USA 104: 15081-15086, 2007.

7. Shi Y, Hutchins W, Ogawa H, Chang CC, Pritchard KA Jr, Zhang C, Khampang P, Lazar J, Jacob HJ, Rafiee P and Baker JE: Increased resistance to myocardial ischemia in the Brown Norway vs. Dahl S rat: Role of nitric oxide synthase and Hsp90. J Mol Cell Cardiol 38: 625-635, 2005.
8. An J, Du J, Wei N, Xu H, Pritchard KA Jr and Shi Y: Role of tetrahydrobiopterin in resistance to myocardial ischemia in Brown Norway and Dahl S rats. Am J Physiol Heart Circ Physiol 297: H1783-H1791, 2009.

9. Xie L, Talukder MA, Sun J, Varadharaj S and Zweier JL: Liposomal tetrahydrobiopterin preserves eNOS coupling in the post-ischemic heart conferring in vivo cardioprotection. J Mol Cell Cardiol 86: 14-22, 2015

10. Kawahara K, Takase M and Yamauchi Y: Increased vulnerability to ischemia/reperfusion-induced ventricular tachyarrhythmias by pre-ischemic inhibition of nitric oxide synthase in isolated rat hearts. Cardiovasc Pathol 12: 49-56, 2003.

11. Baotic I, Weihrauch D, Procknow J, Vasquez-Vivar J, Ge ZD, Sudhakaran S, Warltier DC and Kersten JR: Isoflurane favorably modulates guanosine triphosphate cyclohydrolase-1 and endothelial nitric oxide synthase during myocardial ischemia and reperfusion injury in rats. Anesthesiology 123: 582-589, 2015.

12. Guerrero-Orriach JL, Escalona Belmonte JJ, Ramirez Fernandez A, Ramirez Aliaga M, Rubio Navarro $M$ and Cruz Manas J: Cardioprotection with halogenated gases: How does it occur? Drug Des Devel Ther 11: 837-849, 2017.

13. Novalija E, Varadarajan SG, Camara AK, An J, Chen Q, Riess ML, Hogg N and Stowe DF: Anesthetic preconditioning: Triggering role of reactive oxygen and nitrogen species in isolated hearts. Am J Physiol Heart Circ Physiol 283: H44-H52, 2002.

14. Amour J, Le Manach YL, Borel M, Lenfant F, Nicolas-Robin A, Carillion A, Ripart J, Riou B and Langeron O: Comparison of single-use and reusable metal laryngoscope blades for orotracheal intubation during rapid sequence induction of anesthesia: A multicenter cluster randomized study. Anesthesiology 112: 325-332, 2010.

15. Stowe DF, Gadicherla AK, Zhou Y, Aldakkak M, Cheng Q, Kwok WM, Jiang MT, Heisner JS, Yang M and Camara AK: Protection against cardiac injury by small $\mathrm{Ca}(2+)$-sensitive $\mathrm{K}(+)$ channels identified in guinea pig cardiac inner mitochondrial membrane. Biochim Biophys Acta 1828: 427-442, 2013.

16. Chatterjee A, Black SM and Catravas JD: Endothelial nitric oxide (NO) and its pathophysiologic regulation. Vascul Pharmacol 49: 134-140, 2008.

17. Thomas SR, Chen K and Keaney JF Jr: Oxidative stress and endothelial nitric oxide bioactivity. Antioxid Redox Signal 5: 181-194, 2003.

18. Vasquez-Vivar J, Kalyanaraman B, Martasek P, Hogg N, Masters BS, Karoui H, Tordo P and Pritchard KA Jr: Superoxide generation by endothelial nitric oxide synthase: The influence of cofactors. Proc Natl Acad Sci USA 95: 9220-9225, 1998.

19. Hemmens B and Mayer B: Enzymology of nitric oxide synthases. Methods Mol Biol 100: 1-32, 1998.

20. Leucker TM, Ge ZD, Procknow J, Liu Y, Shi Y, Bienengraeber M, Warltier DCand KerstenJR:Impairment of endothelial-myocardial interaction increases the susceptibility of cardiomyocytes to ischemia/reperfusion injury. PLoS One 8: e70088, 2013.

21. Vasquez-Vivar J, Kalyanaraman B and Martasek P: The role of tetrahydrobiopterin in superoxide generation from eNOS: Enzymology and physiological implications. Free Radic Res 37: 121-127, 2003.

22. Gorren AC, List BM, Schrammel A, Pitters E, Hemmens B, Werner ER, Schmidt K and Mayer B: Tetrahydrobiopterin-free neuronal nitric oxide synthase: Evidence for two identical highly anticooperative pteridine binding sites. Biochemistry 35: 16735-16745, 1996.

23. Moens AL and Kass DA: Tetrahydrobiopterin and cardiovascular disease. Arterioscler Thromb Vasc Biol 26: 2439-2444, 2006.

24. Tiefenbacher CP, Lee CH, Kapitza J, Dietz V and Niroomand F: Sepiapterin reduces postischemic injury in the rat heart. Pflugers Arch 447: 1-7, 2003.

25. Liu Y, Baumgardt SL, Fang J, Shi Y, Qiao S, Bosnjak ZJ, Vasquez-Vivar J, Xia Z, Warltier DC, Kersten JR and Ge ZD: Transgenic overexpression of GTP cyclohydrolase 1 in cardiomyocytes ameliorates post-infarction cardiac remodeling. Sci Rep 7: 3093, 2017.

26. Couto GK, Paula SM, Gomes-Santos IL, Negrao CE and Rossoni LV: Exercise training induces eNOS coupling and restores relaxation in coronary arteries of heart failure rats. Am J Physiol Heart Circ Physiol 314: H878-H887, 2018.

27. Vladic N, Ge ZD, Leucker T, Brzezinska AK, Du JH, Shi Y, Warltier DC, Pratt PF Jr and Kersten JR: Decreased tetrahydrobiopterin and disrupted association of Hsp90 with eNOS by hyperglycemia impair myocardial ischemic preconditioning. Am J Physiol Heart Circ Physiol 301: H2130-H2139, 2011. 